\title{
Alcohol and disease activity in axial spondyloarthritis: a cross- sectional study
}

\author{
Sizheng Zhao ${ }^{1,2}$ (D) Daniel Thong ${ }^{1} \cdot$ Stephen J. Duffield ${ }^{2}$. David Hughes ${ }^{3} \cdot$ Nicola J. Goodson $^{1,2}$
}

Received: 14 December 2017 / Accepted: 2 January 2018 / Published online: 10 January 2018

(c) The Author(s) 2018. This article is an open access publication

\begin{abstract}
The objective of this study was to explore associations between alcohol consumption and disease activity in axial spondyloarthritis (axSpA). We conducted a cross-sectional study of axSpA participants meeting the ASAS criteria. Associations between self-reported current alcohol use and disease activity (BASDAI, spinal pain, ASDAS), functional impairment (BASFI), and quality of life were explored using multivariable linear models, adjusting for age, gender, symptom duration, use of TNF inhibition therapy, smoking, deprivation, and anxiety and depression (A\&D). Within alcohol drinkers, effect of increased alcohol intake (defined as $>14$ units/week) was explored with moderate drinking ( $\leq 14$ units/week) as reference. The study cohort comprised 229 axSpA patients and $76 \%$ were male with mean age 46.5 years $(S D \pm 13.8)$. Alcohol drinking was reported by $64 \%$, with a median of 6 units per week among drinkers. Compared with non-drinkers, drinkers had lower BASDAI $(\beta=-0.83 ; 95 \% \mathrm{CI}-1.49,-0.17)$, ASDAS $(\beta=-0.36 ; 95 \% \mathrm{CI}-0.66,-0.05)$ and BASFI $(\beta=-1.40 ; 95 \%$ $\mathrm{CI}-2.12,-0.68)$. These associations were in contrast to, and independent of, the detrimental effects of smoking, depression, and deprivation. Subgroup analysis in alcohol drinkers did not reveal significant associations between disease severity and increased alcohol intake. Stratified analyses by smoking revealed that in never-smokers without depression, alcohol was associated with greater reduction in disease activity: BASDAI $(\beta=-1.69 ; 95 \% \mathrm{CI}-2.93,-0.45)$, ASDAS $(\beta=-0.60 ; 95 \%$ $\mathrm{CI}-1.18,-0.02)$. Favourable axSpA disease activity and function were observed in association with alcohol consumption in this cross-sectional study. Longitudinal study is required to explore whether this relationship is due to biological effects of alcohol on disease process or disease-associated behaviour modification.
\end{abstract}

Keywords Axial spondyloarthritis · Ankylosing spondylitis · Alcohol · Disease activity · Functional impairment · Quality of life

\section{Introduction}

Axial spondyloarthritis (axSpA) is a chronic inflammatory disease predominantly affecting the axial skeleton. Potentially modifiable environmental risk factors for increased disease activity and severity have received much research interest. There is emerging evidence that cigarette smoking is associated with a more severe disease phenotype in axSpA

Nicola J. Goodson

ngoodson@liverpool.ac.uk

1 Department of Academic Rheumatology, Aintree University Hospital, Liverpool, UK

2 Musculoskeletal Biology I, Institute of Ageing and Chronic Disease, University of Liverpool, Liverpool, UK

3 Department of Biostatistics, Institute of Translational Medicine, University of Liverpool, Liverpool, UK
$[1,2]$. Some studies have also suggested that dietary modification may reduce disease activity in AS [3]. However, the impact of alcohol consumption on axSpA disease activity and functional impairment has not been extensively studied, despite one study identifying an association between alcohol intake with radiographic damage in early disease [4].

Moderate alcohol intake has often been reported to beneficial for health [5] and is associated with reduced CRP in the general population [6]. In rheumatoid arthritis, alcohol consumption is inversely associated with risk [7], severity [8], extra-articular manifestations [9], and radiographic progression [10]. However, in psoriasis and psoriatic arthritis the converse in observed: alcohol consumption was associated with increased disease susceptibility independent of smoking $[11,12]$. Therefore, the role of alcohol in inflammatory diseases, and particularly in axSpA, is far from clear. Lifestyle behaviours such as alcohol intake and smoking are 
frequently associated with demography and socioeconomic factors as well as psychological status. In any study examining impact of alcohol on inflammatory disease, adjustments for these potential confounding variables need to be considered. The aim of this study was to explore associations between alcohol consumption and disease activity and function in axSpA.

\section{Methods}

Consecutive axSpA patients attending a tertiary referral spondyloarthritis service in Liverpool, UK, were recruited between 2010 and 2015. Patients were included if they fulfilled the ASAS criteria for axial SpA. Clinical data were collected at the initial clinical assessment including: age, gender, body mass index (BMI), smoking status, pack-years; meeting modified New York criteria for AS, symptom duration, duration since diagnosis, HLA-B27, extra-axial features; current use of NSAIDs, and tumour necrosis factor inhibition therapy (TNFi). Socioeconomic status was approximated using index of multiple deprivation (IMD) based on post-codes [13]. Decile 1 relates to the top $10 \%$ most deprived areas, whilst decile 10 represents the least deprived areas.

At the initial clinical assessment, participants reported frequency of drinking and estimated their average weekly alcohol consumption in units, with 1 unit is equal to $10 \mathrm{ml}$ or $8 \mathrm{~g}$ of pure alcohol. They were categorised as drinkers or non-drinkers (including previous drinkers that have stopped for $>1$ year). "Occasional" and "social" drinkers were classed as drinkers. Alcohol intake was used to divide drinkers into two groups, which, in this study, were labelled "moderate" ( $\leq 14$ units/week) or "heavy" drinkers (>14 units/week), for both genders as per current UK Department of Health guidelines [14]. This dichotomisation was performed, because retrospective patient estimates of units/week were unlikely to be accurate and were often in multiples of 7 .

Disease activity and functional status were assessed using numeric rating scale versions of the Bath AS Disease Activity Index (BASDAI), spinal pain, and Bath AS Functional Index (BASFI). AS disease activity score (ASDAS) was introduced after study commencement and was, therefore, missing at random. Quality of life was assessed by EuroQol (EQ5D-3L) using UK value sets and overall health status visual analogue scale (EQ-VAS). EQ5D index is anchored at 1 when there are no problems in all domains, and 0 as death [15]. Anxiety and depression (A\&D) domain was extracted from EQ5D-3L and treated as a binomial variable recording the presence or absence of any self-reported A\&D. Blood samples were taken on the day of assessment for ESR and CRP.

\section{Analysis}

Statistical analyses were performed using Stata13. Comparison of patient and disease characteristics between drinking status and quantity were performed using Student's $t$ test for normally distributed, Mann-Whitney $U$ test for non-normal, and Chi-squared or Fisher's exact test for categorical variables.

Multivariable linear models were generated to explore the association between drinking status as the dichotomous independent variable and each measure of disease activity in turn. Models were adjusted for age, gender, symptom duration, use of TNFi, ever-smoking, IMD, and A\&D. Additional models were generated to examine interactions between all covariates. Within drinkers, multivariable linear models with the above covariates were used to compare heavy drinkers to those reporting moderate intake. For sensitivity analysis, gender-specific tertiles of units/week were also generated within drinkers to explore intake quantity in multivariable models, using the lowest tertile as the reference group.

EQ5D index could not be adequately transformed and was, therefore, dichotomised by its median and analysed using logistic regression with the above covariates except A\&D. AS diagnosis, use of NSAIDs, and HLA-B27 did not improve the model (assessed by likelihood ratio test) and were, therefore, not included. ESR and CRP were log-transformed prior to regression. Missing data were not imputed.

This study complies with the Declaration of Helsinki and was approved by the UK Research Ethics Committee $(15 / \mathrm{LO} / 1519)$.

\section{Results}

The study recruited 238 participants with established axSpA of whom 229 (96\%) had complete alcohol history. The cohort was predominantly male $(76 \%)$ with mean age of 46.5 years $(S D \pm 13.8)$ and $83 \%$ met modified New York criteria for AS. The median symptom duration was 17.1 years [interquartile range (IQR) 8.4, 29.3]. HLA-B27 was measured in $141(62 \%)$ and of these $60 \%$ were positive. TNFi treatment was used by 76 (33\%). The median BASDAI was 5.7 (IQR 3.3, 7.6) and BASFI 5.7 (IQR 3.3, 7.6). ASDAS was available for $182(79 \%)$ participants with mean of 2.7 $(\mathrm{SD} \pm 1.1)$. Alcohol drinking was reported by 147 (64\%). Amongst drinkers, the median quantity was 6 units per week (IQR 2, 20). Only two participants described themselves as ex-drinkers. There were no differences in participant or disease characteristics between those with alcohol history and the minority without (data not shown). 
Table 1 Patient and disease characteristics compared between alcohol status and quantity categories

\begin{tabular}{|c|c|c|c|c|c|c|}
\hline & No alcohol & Alcohol drinker & $P$ value & $\begin{array}{l}\text { Moderate drinking } \\
\text { ( } \leq 14 \text { units/week) }\end{array}$ & $\begin{array}{l}\text { Heavy drinking } \\
\text { (>14 units/week) }\end{array}$ & $P$ value \\
\hline Number of patients & $82(36 \%)$ & $147(64 \%)$ & & $104(71 \%)$ & $43(29 \%)$ & \\
\hline Age & $45.5(14.3)$ & $47.0(13.6)$ & 0.409 & $46.1(14.0)$ & $49.4(12.4)$ & 0.177 \\
\hline Male gender & $51(62 \%)$ & $122(83 \%)$ & $<0.001$ & $79(76 \%)$ & $43(100 \%)$ & $<0.001$ \\
\hline $\mathrm{BMI}^{\mathrm{a}}$ & $28.6(4.7)$ & $28.0(5.7)$ & 0.494 & $27.6(5.8)$ & $28.9(5.3)$ & 0.261 \\
\hline IMD decile & $2[1,5]$ & $2[1,6]$ & 0.143 & $3[1,6]$ & $2[1,5]$ & 0.889 \\
\hline AS diagnosis & $68(83 \%)$ & $122(83 \%)$ & 0.990 & $85(82 \%)$ & $37(86 \%)$ & 0.526 \\
\hline HLA-B27 status ${ }^{\mathrm{a}}$ & $30(53 \%)$ & $55(65 \%)$ & 0.126 & $40(65 \%)$ & $15(68 \%)$ & 0.756 \\
\hline Symptom duration (years) & $16.2[6.5,29.3]$ & $17.2[9.1,29.6]$ & 0.391 & $16.8[8.4,29.2]$ & $20.4[10.5,32.5]$ & 0.317 \\
\hline Diagnosis duration (years) & $4.0[0.7,12.6]$ & $5.2[0.9,15.2]$ & 0.177 & $3.6[0.8,14.4]$ & $8.0[1.7,17.6]$ & 0.087 \\
\hline Ever-smoking & $37(45 \%)$ & $70(48 \%)$ & 0.717 & $42(40 \%)$ & $28(65 \%)$ & 0.006 \\
\hline Pack-years & $0[0,15]$ & $0[0,20]$ & 0.953 & $\mathbf{0}[\mathbf{0}, \mathbf{1 0}]$ & $10[0,20]$ & 0.008 \\
\hline Self-reported A\&D & $43(57 \%)$ & $71(52 \%)$ & 0.505 & $46(47 \%)$ & $25(64 \%)$ & 0.070 \\
\hline BASDAI & $6.7[3.7,8.3]$ & $5.3[3,7.2]$ & 0.002 & $5.3[2.9,7.2]$ & $5.1[3.1,7.2]$ & 0.914 \\
\hline Spinal pain & $7.0[4.0,9.0]$ & $5.0[3.0,8.0]$ & 0.008 & $5[3,8]$ & $6[2,8]$ & 0.888 \\
\hline $\mathrm{ASDAS}^{\mathrm{a}}$ & $2.93(1.22)$ & $2.57(1.09)$ & 0.040 & $2.49(1.08)$ & $2.78(1.10)$ & 0.199 \\
\hline BASFI & $6.5[4.2,8.4]$ & $5.0[2.1,7.3]$ & 0.001 & $4.9[2,7.1]$ & $5.7[2.4,7.5]$ & 0.355 \\
\hline $\mathrm{CRP}(\mathrm{mg} / \mathrm{L})^{\mathrm{a}}$ & $3[1,10]$ & $3[1,7]$ & 0.582 & $3[1,7]$ & $3[1,6]$ & 0.434 \\
\hline $\operatorname{ESR}(\mathrm{mm} / \mathrm{hr})^{\mathrm{a}}$ & $9[5,23]$ & $8[5,19]$ & 0.412 & $8[5,20]$ & $6.5[2,13.5]$ & 0.113 \\
\hline EQ5D index ${ }^{a}$ & $0.52[0.01,0.69]$ & $0.59[0.09,0.76]$ & 0.068 & $0.68[0.16,0.80]$ & $0.16[-0.07,0.76]$ & 0.008 \\
\hline EQ-VAS $^{\mathrm{a}}$ & $53.1(21.9)$ & $43.7(25.0)$ & 0.008 & $41.8(2.83)$ & $46.2(3.30)$ & 0.315 \\
\hline Peripheral joint involvement & $20(24 \%)$ & $32(23 \%)$ & 0.751 & $23(22 \%)$ & $9(23 \%)$ & 0.924 \\
\hline Psoriasis & $14(17 \%)$ & $23(16 \%)$ & 0.779 & $17(16 \%)$ & $6(14 \%)$ & 0.716 \\
\hline Uveitis & $18(22 \%)$ & $45(31 \%)$ & 0.159 & $31(30 \%)$ & $14(33 \%)$ & 0.742 \\
\hline IBD & $7(9 \%)$ & $14(10 \%)$ & 0.804 & $9(9 \%)$ & $5(12 \%)$ & 0.552 \\
\hline TNFi & $27(33 \%)$ & $49(33 \%)$ & 0.950 & $29(28 \%)$ & $20(47 \%)$ & 0.029 \\
\hline NSAID & $51(62 \%)$ & $106(72 \%)$ & 0.121 & $76(73 \%)$ & $30(70 \%)$ & 0.684 \\
\hline
\end{tabular}

Statistically significant results are presented in bold

Data are presented in $n(\%)$, mean (SD), and median [IQR]

$A \& D$ anxiety and depression, IMD index of multiple deprivation, $A S D A S$ Ankylosing Spondylitis Disease Activity Score, $E Q$ EuroQoL, $I B D$ inflammatory bowel disease, TNFi TNF inhibition therapy, NSAID non-steroidal anti-inflammatory drugs

${ }^{a}$ BMI complete data in 182; HLAB27 status known in 141; ASDAS in 182; CRP in 222; ESR in 221, EuroQol scores, and A\&D in 213

Participant and disease characteristics are shown in Table 1. Male participants were more likely to report drinking than females. No significant differences were seen between alcohol status and age, symptom duration, and proportion of AS, BMI, or extra-axial features, including psoriasis. There was no observed association between drinking and smoking. Most markers of disease severity were significantly worse in non-drinkers.

Compared to moderate drinkers, heavy drinkers were more frequently male, were more likely to be smokers, and had greater pack-year smoking exposure. There were no differences in age and socioeconomic status. However, heavy drinkers reported significantly worse quality of life and in addition were more likely to be using TNFi therapy at the time of assessment. Alcohol status and quantity were not associated with IMD. However, ever-smoking
$(P=0.01)$ and $\mathrm{A} \& \mathrm{D}(P=0.01)$ were both associated with higher deprivation.

There was no association between alcohol drinking and A\&D $(P=0.51)$, but there was a trend towards more A\&D in heavy drinkers (64 vs 47\%, $P=0.07$ ). Ever-smokers more commonly reported A\&D than never-smokers (54 vs 46\%, $P=0.03)$. There was no association between any alcohol use and smoking $(P=0.72)$. However, heavy drinkers more frequently reported cigarette smoking than moderate drinkers ( 75 vs $40 \%, P=0.01)$.

In multi-adjusted linear models without interaction terms, alcohol drinking was associated with lower disease activity (BASDAI $\beta=-0.83,95 \%$ CI $-1.49,-0.17$; ASDAS $\beta=-0.36,95 \% \mathrm{CI}-0.66,-0.05)$ and functional impairment (BASFI $\beta=-1.40,95 \%$ CI $-2.12,-0.68$ ) (Table 2). As covariates in this model, A\&D was associated with 
Table 2 Multivariable linear models comparing disease activity, functional impairment, and quality of life between alcohol drinkers and nondrinkers, adjusting for age, gender, symptom duration, use of TNFi, ever-smoking, IMD, and A\&D

\begin{tabular}{|c|c|c|c|c|c|}
\hline & \multirow[t]{2}{*}{$\begin{array}{l}\beta \text { coefficient }(95 \% \mathrm{CI}) \\
\text { without interaction terms }\end{array}$} & \multicolumn{2}{|c|}{$\beta$ coefficient $(95 \% \mathrm{CI})$ in never-smokers ${ }^{\mathrm{b}}$} & \multicolumn{2}{|c|}{$\begin{array}{l}\beta \text { coefficient }(95 \% \mathrm{CI}) \text { in ever-smokers if } \mathrm{A} \& \mathrm{D} \\
\text { is absent }^{\mathrm{b}}\end{array}$} \\
\hline & & if $A \& D$ & if $A \&$ & if $A \& D$ is present & if $A \& D$ is absent \\
\hline BASDAI & $-0.83(-1.49,-0.17)$ & $0.58(-0.66,1.83)$ & $-1.69(-2.93,-0.45)$ & 0.32( & $-1.64(-3.24,-0.05)$ \\
\hline ASDAS & $-0.36(-0.66,-0.05)$ & $0.24(-0.36,0.84)$ & $-0.60(-1.18,-0.02)$ & $.32,1.02)$ & $-0.71(-1.41,-0.01)$ \\
\hline Spinal pain & $-0.79(-1.56,-0.02)$ & $0.44(-0.97,1.84)$ & $-2.11(-3.51,-0.71)$ & $.79,2.98)$ & $-0.44(-2.37,1.49)$ \\
\hline BASFI & $-1.40(-2.12,-0.68)$ & $-0.18(-1.52,1.15)$ & $-2.25(-3.57,-0.92)$ & $0.14(-1.56,1.84)$ & $-2.46(-4.20,-0.73)$ \\
\hline EQ-VAS & $-7.46(-13.6,-1.34)$ & $11.4(-1.18,24.0)$ & $-13.9(-26.4,-1.36)$ & $10.8(-2.98,24.6)$ & $-7.84(-22.0,6.33)$ \\
\hline $\operatorname{Ln}(\mathrm{CRP})$ & $-0.19(-0.56,0.19)$ & $-0.07(-0.78,0.63)$ & $-0.13(-0.84,0.57)$ & $0.26(-0.67,1.19)$ & $-0.55(-1.51,0.42)$ \\
\hline $\operatorname{Ln}(\mathrm{ESR})$ & $-0.15(-0.44,0.15)$ & $-0.10(-0.61,0.41)$ & $-0.06(-0.57,0.45)$ & $0.53(-0.22,1.29)$ & $-0.11(-0.88,0.67)$ \\
\hline
\end{tabular}

Statistically significant results are presented in bold

Total sample size for each regression model was 213 for BASDAI, BASFI and spinal pain, 181 for ASDAS, 206 EQ-VAS, 179 CRP, and 204 ESR

BASDAI Bath ankylosing spondylitis disease activity index, ASDAS Ankylosing Spondylitis Disease Activity Score, BASFI Bath AS functional index, $E Q-V A S$ EuroQol visual analogue scale, $T N F i$ TNF inhibitor, $I M D$ index of multiple deprivation, $A \& D$ anxiety and depression

${ }^{a}$ Model: $y=$ alcohol + age + gender + symptom duration + TNFi + eversmoking + IMD + A\&D

${ }^{\mathrm{b}}$ Model: $y=$ alcohol + age + gender + symptom duration + TNFi $+\mathrm{IMD}+\mathrm{A} \& \mathrm{D}+$ alcohol*A $\& \mathrm{D}$

higher disease activity assessed by all markers except CRP, whilst ever-smoking was associated with higher ASDAS, ESR, and CRP (Table 3). Deprivation (IMD) was associated with worse BASFI, quality of life, and ESR.

Alcohol status showed significant interactions with both ever-smoking and A\&D in this model. Stratification by eversmoking revealed an interaction between alcohol and A\&D which was significant in ever-smokers but not in never-smokers. Within the never-smoking subgroup, a strong association between alcohol and reduced disease severity was observed (Table 2). Ever-smokers who consumed alcohol reported reduced BASDAI and BASFI, only in the absence of A\&D.

Disease activity, functional impairment, and quality of life were not significantly different between heavy and moderate drinking. Sensitivity analyses using gender-specific tertiles also did not demonstrate an association between intake quantity and disease outcome measures. Multivariable logistic models of the dichotomised EQ5D index were not associated with alcohol status or quantity (data not shown).

\section{Discussion}

Study of this cross-sectional axSpA cohort demonstrated an inverse association between alcohol drinking and disease activity and functional impairment. The association between alcohol and disease activity was in contrast to, and independent of, the detrimental effects of smoking, depression, and deprivation. A lower BASDAI of 0.8 to 1.6 is clinically relevant, as $\geq 2$-unit improvement is considered a response to TNFi therapy [16]. In never-smokers, alcohol was associated with still greater reduction in disease activity.
Table 3 Effect sizes of anxiety and depression, ever-smoking, and index of deprivation as covariates in multivariable models of alcohol and disease activity, functional impairment, and quality of life

\begin{tabular}{|c|c|c|c|}
\hline & Anxiety and depression & Ever-smoking & Index of multiple deprivation \\
\hline BASDAI & $1.68(1.01,2.31)$ & $0.62(-0.02,1.26)$ & $-0.07(-0.19,0.04)$ \\
\hline ASDAS & $0.75(0.44,1.06)$ & $0.51(0.21,0.81)$ & $-0.03(-0.08,0.02)$ \\
\hline Spinal pain & $1.70(0.94,2.45)$ & $0.47(-0.28,1.22)$ & $-0.12(-0.26,0.01)$ \\
\hline BASFI & $1.78(1.08,2.48)$ & $0.51(-0.19,1.20)$ & $-0.14(-0.27,-0.02)$ \\
\hline EQ-VAS & $19.9(14.0,25.8)$ & $2.22(-3.62,8.07)$ & $-1.83(-2.88,-0.79)$ \\
\hline $\operatorname{Ln}(\mathrm{CRP})$ & $0.30(-0.07,0.66)$ & $0.45(0.08,0.81)$ & $0.05(-0.02,0.11)$ \\
\hline $\operatorname{Ln}(\mathrm{ESR})$ & $0.32(0.03,0.61)$ & $0.37(0.08,0.65)$ & $0.07(0.02,0.12)$ \\
\hline
\end{tabular}

Statistically significant results are presented in bold

Total sample size for each regression model was 213 for BASDAI, BASFI, and spinal pain, 181 for ASDAS, 206 EQ-VAS, 179 CRP, and 204 ESR

$B A S D A I$ Bath ankylosing spondylitis disease activity index, ASDAS Ankylosing Spondylitis Disease Activity Score, BASFI Bath AS functional index, EQ-VAS EuroQol visual analogue scale 
In ever-smokers, A\&D appears to counteract the reduced disease activity seen with alcohol use. This is interesting, since alcohol and depression each increase the risk of the other and yet appear to have opposing effects on disease activity in axSpA patients.

Only two studies have explored alcohol consumption in axSpA. In a cross-sectional study, Zhang et al. focused on BASDAI (dichotomised at 4) and found increased odds of high BASDAI in moderate drinkers compared to non-drinkers, but analyses did not account for treatment or A\&D and was not significant when adjusted for smoking [17]. Blachier et al. reported significantly increased odds of drinking in axSpA patients with radiographic changes at the sacroiliac joints and/or vertebrae [4]. The covariates were not explicit stated but did not include A\&D, and no comments were made on disease activity.

In addition to more frequently reporting alcohol drinking, male patients were also more frequently categorised as heavy drinkers, which is consistent with population studies. Male gender in axSpA studies is usually associated with more severe disease. The finding that disease activity and functional impairment were both lower in alcohol drinkers, despite this male gender preponderance, is interesting.

Within the subgroup of participants reporting alcohol use, increasing tertiles of alcohol exposure was not associated with improved axSpA disease measures. This lack of dose-response effect is less suggestive of a biological protective effect from alcohol consumption. However, the relationship between alcohol intake and inflammation in population studies typically follows a U-shaped distribution with modest intake conferring protection which is lost at higher levels of alcohol consumption [18]. It is possible that the lack of association between axSpA disease measures with amount of alcohol consumed in this study could be due to the same phenomenon. Alcohol has been shown to suppress production of proinflammatory cytokines such as TNF and IL6 in human cell models, and moderate alcohol consumption is associated with lower levels of CRP, IL6, and soluble TNF receptors in healthy adults [19, 20]. Further study in larger disease population is required to explore this in more detail.

No associations between alcohol drinking and any extraarticular disease manifestations were seen in this study. This differs from the previous studies which have highlighted the association between alcohol use and disease susceptibility to psoriasis and psoriatic arthritis $[11,12]$.

There was no difference in self-reported A\&D symptoms between drinkers and non-drinkers. This was not consistent with other studies where non-drinkers were less likely to develop A\&D [21]. Self-reported A\&D was significantly associated with worse disease activity and functional impairment with effect sizes larger than smoking or deprivation. There was no association between ever-smoking and drinking. However, heavy drinkers were more frequently smokers, which is consistent with general population studies showing a dose-dependent relationship between alcohol use and smoking [22]. Interestingly, ever-smoking was associated with only ASDAS, CRP, and ESR, but none of the other more subjective disease activity markers in multivariable models. Smoking's previous reported effect on the Bath indices may be explained by confounding factors accounted for in this study. There was no difference in deprivation between alcohol status. However, increasing deprivation was independently associated with worse BASFI and quality of life. Despite the detrimental effects of smoking, depression, and deprivation, we observed lower disease activity and functional impairment in drinkers when these factors were adjusted for.

Studies of alcohol's impact on health outcomes often report unexpected and conflicting results [23]. These inconsistencies may be explained by several methodological limitations. First, there are many confounders that should be considered. Alcohol drinking, depression, smoking, and socioeconomic status (SES) are interlinked and each have well-known effects on disease severity. The strength of this study is that these factors were adjusted for. However, this study cohort was recruited from an area with generally high levels of deprivation. Future studies should recruit a more mixed population. Second, many alcohol studies have been criticised for the heterogeneity of their non-drinker comparator, which often included occasional drinkers [23]. For this reason, two common responses-social and occasional drinking-were classified as drinkers in this study to provide a more homogenous comparator group. However, we were unable to perform sensitivity analyses of different categorisations. Third, it has been shown that alcohol intake is underestimated in surveys due to social desirability bias [23]. This study may be more susceptible to this bias given information was collected during clinical consultations. This may explain the low number of ex-drinkers. Like most alcohol studies, we focused on volume rather than frequency or quality. Future studies should distinguish patterns of drinking (such as binge-drinking) and also type of alcohol consumed (such as spirits, wines, beers). Wines are of particular interest as they are rich in anti-inflammatory polyphenols, such as resveratrol. These compounds inhibit TNF-induced nuclear factor kappa B (NF- $\mathrm{kB})$ activation and may have protective properties in inflammatory arthritides [24].

The main limitation of this study was its cross-sectional design, which does not allow change in drinking behaviour to be considered. First, it is possible that those with more challenging disease activity may modify their behaviour and reduce alcohol intake to improve coping mechanisms. Second, studies have shown that measuring alcohol consumption during one time period in a participant's life may be unreliable [6]. Repeated assessments of alcohol intake should be considered in future studies. 
In conclusion, this study is the first to report that alcohol consumption is associated with reduced measures of axSpA disease severity than in non-drinkers. Further studies are needed to establish a causal link. These results cannot be used to endorse or promote the use of alcohol as both scientific evidence and guidelines are increasingly downplaying the previously held belief that moderate alcohol is beneficial. Furthermore, alcohol intake of three or more units per day is a recognised risk factor for osteoporosis [25], which is an important complication in axSpA.

Acknowledgements We thank our patients for taking part in this research.

Funding No funding was received for this study.

\section{Compliance with ethical standards}

Conflict of interest The authors declare no conflicts of interest.

Ethical approval All procedures performed in studies involving human participants were in accordance with the ethical standards of the institutional and/or national research committee and with the 1964 Helsinki declaration and its later amendments or comparable ethical standards.

Informed consent Informed consent was obtained from all individual participants included in the study.

Open Access This article is distributed under the terms of the Creative Commons Attribution 4.0 International License (http://creativecommons.org/licenses/by/4.0/), which permits unrestricted use, distribution, and reproduction in any medium, provided you give appropriate credit to the original author(s) and the source, provide a link to the Creative Commons license, and indicate if changes were made.

\section{References}

1. Zhao S, Challoner B, Khattak M, Moots RJ, Goodson NJ (2017) Increasing smoking intensity is associated with increased disease activity in axial spondyloarthritis. Rheumatol Int 37(2):239-244. https://doi.org/10.1007/s00296-016-3590-4

2. Mattey DL, Dawson SR, Healey EL, Packham JC (2011) Relationship between smoking and patient-reported measures of disease outcome in ankylosing spondylitis. J Rheumatol 38(12):26082615. https://doi.org/10.3899/jrheum.110641

3. Ebringer A, Rashid T, Wilson C (2016) Ankylosing spondylitis, Klebsiella and the low-starch diet. In: Nibali L, Henderson B (eds) The human microbiota and chronic disease: dysbiosis as a cause of human pathology. Wiley, NJ. https://doi. org/10.1002/9781118982907.ch20

4. Blachier M, Canoui-Poitrine F, Dougados M, Lethuaut A, Fautrel B, Ferkal S, Le Corvoisier P, Farrenq V, Poulain C, Ghaleh B, Bastuji-Garin S, Claudepierre P (2013) Factors associated with radiographic lesions in early axial spondyloarthritis. Results from the DESIR cohort. Rheumatology 52(9):1686-1693. https://doi. org/10.1093/rheumatology/ket207

5. Thun MJ, Peto R, Lopez AD, Monaco JH, Henley SJ, Heath CW Jr, Doll R (1997) Alcohol consumption and mortality among middle-aged and elderly US adults. N Engl J Med 337(24):17051714. https://doi.org/10.1056/NEJM199712113372401

6. Bell S, Mehta G, Moore K, Britton A (2017) Ten-year alcohol consumption typologies and trajectories of $\mathrm{C}$-reactive protein, interleukin- 6 and interleukin- 1 receptor antagonist over the following 12 years: a prospective cohort study. J Intern Med 281(1):75-85. https://doi.org/10.1111/joim.12544

7. Kallberg H, Jacobsen S, Bengtsson C, Pedersen M, Padyukov L, Garred P, Frisch M, Karlson EW, Klareskog L, Alfredsson L (2009) Alcohol consumption is associated with decreased risk of rheumatoid arthritis: results from two Scandinavian case-control studies. Ann Rheum Dis 68(2):222-227. https://doi.org/10.1136/ $\operatorname{ard} .2007 .086314$

8. Maxwell JR, Gowers IR, Moore DJ, Wilson AG (2010) Alcohol consumption is inversely associated with risk and severity of rheumatoid arthritis. Rheumatology 49(11):2140-2146. https:// doi.org/10.1093/rheumatology/keq202

9. Kim SK, Park SH, Shin IH, Choe JY (2008) Anti-cyclic citrullinated peptide antibody, smoking, alcohol consumption, and disease duration as risk factors for extraarticular manifestations in Korean patients with rheumatoid arthritis. J Rheumatol 35(6):995-1001

10. Nissen MJ, Gabay C, Scherer A, Finckh A, Arthritis SCQMPiR (2010) The effect of alcohol on radiographic progression in rheumatoid arthritis. Arthritis Rheum 62(5):1265-1272. https://doi. org/10.1002/art.27388

11. Zhu KJ, Zhu CY, Fan YM (2012) Alcohol consumption and psoriatic risk: a meta-analysis of case-control studies. J Dermatol 39(9):770-773. https://doi.org/10.1111/j.1346-8138.2012.01577.x

12. Wu S, Cho E, Li WQ, Han J, Qureshi AA (2015) Alcohol intake and risk of incident psoriatic arthritis in women. J Rheumatol 42(5):835-840. https://doi.org/10.3899/jrheum.140808

13. Department for Communities and Local Government (2015) English indices of deprivation 2015. http://www.gov.uk/government/ statistics/english-indices-of-deprivation-2015. Accessed Dec 2017

14. Department of Health (2016) Alcohol guidelines review-report from the guidelines development group to the UK Chief Medical Officers. https://www.gov.uk/government/uploads/system/ uploads/attachment_data/file/545739/GDG_report-Jan2016.pdf. Accessed Dec 2017

15. van Reene M, Oppe M (2015) EQ-5D-3L user guide v5.1. http:// www.euroqol.org/fileadmin/user_upload/Documenten/PDF/Folders_Flyers/EQ-5D-3L_UserGuide_2015.pdf. Accessed Dec 2017

16. National Institute for Health and Care Excellence (2016) TNFalpha inhibitors for ankylosing spondylitis and non-radiographic axial spondyloarthritis. https://www.nice.org.uk/guidance/ta383/ resources/tnfalpha-inhibitors-for-ankylosing-spondylitis-andnonradiographic-axial-spondyloarthritis-pdf-82602848027077. Accessed Dec 2017

17. Zhang S, Li Y, Xu X, Feng X, Yang D, Lin G (2015) Effect of cigarette smoking and alcohol consumption on disease activity and physical functioning in ankylosing spondylitis: a cross-sectional study. Int J Clin Exp Med 8(8):13919-13927

18. Power C, Rodgers B, Hope S (1998) U-shaped relation for alcohol consumption and health in early adulthood and implications for mortality. Lancet 352(9131):877. https://doi.org/10.1016/ S0140-6736(98)23937-7

19. Lu B, Solomon DH, Costenbader KH, Keenan BT, Chibnik LB, Karlson EW (2010) Alcohol consumption and markers of inflammation in women with preclinical rheumatoid arthritis. Arthritis Rheum 62(12):3554-3559. https://doi.org/10.1002/art.27739

20. Pai JK, Hankinson SE, Thadhani R, Rifai N, Pischon T, Rimm EB (2006) Moderate alcohol consumption and lower levels of inflammatory markers in US men and women. Atherosclerosis 186(1):113-120. https://doi.org/10.1016/j. atherosclerosis.2005.06.037 
21. Haynes JC, Farrell M, Singleton N, Meltzer H, Araya R, Lewis G, Wiles NJ (2008) Alcohol consumption as a risk factor for non-recovery from common mental disorder: results from the longitudinal follow-up of the National Psychiatric Morbidity Survey. Psychol Med 38(3):451-455. https://doi.org/10.1017/ S0033291707002000

22. Falk DE, Yi HY, Hiller-Sturmhofel S (2006) An epidemiologic analysis of co-occurring alcohol and tobacco use and disorders: findings from the National Epidemiologic Survey on Alcohol and Related Conditions. Alcohol Res Health J Natl Inst Alcohol Abuse Alcohol 29(3):162-171
23. Public Health England (2016) The public health burden of alcohol and the effectiveness and cost-effectiveness of alcohol control policies: an evidence review. https://www.gov.uk/government/ uploads/system/uploads/attachment_data/file/583047/alcohol_ public_health_burden_evidence_review.pdf

24. Elmali N, Baysal O, Harma A, Esenkaya I, Mizrak B (2007) Effects of resveratrol in inflammatory arthritis. Inflammation 30(1-2):1-6. https://doi.org/10.1007/s10753-006-9012-0

25. Kanis JA, Oden A, Johansson H, Borgstrom F, Strom O, McCloskey E (2009) FRAX and its applications to clinical practice. Bone 44(5):734-743. https://doi.org/10.1016/j.bone.2009.01.373 